Ryo Suminaga - Yasuhiro Takeshima - Kimiko Yasuda Nobuyuki Shiga - Hajime Nakamura - Masafumi Matsuo

\title{
Non-homologous recombination between Alu and LINE-1 repeats caused a 430-kb deletion in the dystrophin gene: a novel source of genomic instability
}

\begin{abstract}
Although large deletions in the dystrophin gene have been identified in more than two-thirds of Duchenne and Becker muscular dystrophy patients, the molecular mechanisms that lead to the generation of these deletions are largely unknown. Here, Alu and LINE-1 (L1) repetitive elements were shown to be present at one or other of the two ends, respectively, of a $430-\mathrm{kb}$ deletion in the dystrophin gene. The breakpoint of the deletion, which stretches from exons 2 to 7, was defined more precisely by polymerase chain reaction (PCR) walking on introns 1 and 7. Finally, the region containing the breakpoint was amplified as a fragment of more than $10 \mathrm{~kb}$. Sequencing of the deletion endpoint revealed the presence of an Alu sequence in intron $1,25 \mathrm{~kb}$ downstream from the $3^{\prime}$ end of exon 1 that was joined directly to an L1 sequence in intron $7,4.5 \mathrm{~kb}$ downstream from the $3^{\prime}$ end of exon 7 . The deletion was calculated to be $430 \mathrm{~kb}$. To our knowledge, this is a novel recombination event joining non-homologous Alu and L1 repeats, and is the largest known intrachromosomal deletion that is thought to involve repetitive genetic elements. Sequence characteristics around the breakpoint are discussed.
\end{abstract}

Key words Non-homologous recombination · Alu - L1 · Dystrophin gene $\cdot$ Deletion $\cdot$ Duchenne muscular dystrophy

R. Suminaga $\cdot$ N. Shiga $\cdot$ M. Matsuo $(\square)$

Division of Genetics, International Center for Medical Research, Kobe University School of Medicine, 7-5-1 Kusunokicho, Chuo-ku, Kobe 650-0017, Japan

Tel. +81-78-382-5700; Fax +81-78-382-5719

e-mail:matsuo@kobe-u.ac.jp

Y. Takeshima $\cdot$ H. Nakamura

Department of Pediatrics, Kobe University School of Medicine, Kobe, Japan

K. Yasuda

Department of Pediatrics, Chiba University School of Medicine,

Chiba, Japan

\section{Introduction}

Severe Duchenne and mild Becker muscular dystrophies (DMD/BMD) are caused by mutations in the dystrophin gene. The gene occupies $3000 \mathrm{~kb}$ of the $\mathrm{X}$ chromosome and consists of 79 exons. DMD/BMD is the most common inherited muscle disease, affecting 1 in every 3500 boys, and it has been estimated that one-third of DMD/BMD cases result from new mutations in the dystrophin gene (Moser 1984). Two-thirds of DMD/BMD cases have large deletions (Koenig et al. 1987). More than 100 cases have been identified with deletion mutations in the dystrophin gene, and two deletion hot spots have been identified near the $5^{\prime}$ end and in the central region of the gene (Koenig et al. 1987; Forrest et al. 1988). The presence of deletion hot spots in the gene suggests that a particular gene structure within them makes these regions prone to genetic instability. However, the molecular mechanisms underlying the generation of large deletions are largely unknown, because the huge size of the intron hampers molecular analysis of the genetic structure around the breakpoint.

Over one-third of the human genome consists of interspersed repetitive sequences that are primarily degenerate copies of transposable elements. Alu and LINE-1 (L1) sequences, which are present at more than 500,000 locations, are the two most common repeat elements identified on the human genome, representing more than $5 \%$ and $10 \%$ of the human genome, respectively (Smit 1996).

Homologous or non-homologous recombination events are sources of genome instability. Homologous recombination events between Alu repeats and between L1 repeats have been reported in 18 (Deininger and Batzer 1999) and 2 cases (Burwinkel and Kilimann 1998; Segal et al. 1999) of human disorders, respectively. Non-homologous recombination events involving one of these repeat elements at either end of a recombination site have also been reported in a limited number of mutations (Drechsler and Royer-Pokora 1996; Kumatori et al. 1998; Zhang and Zhang 1998; Deininger and Batzer 1999; Morgan et al. 1999). As far as we know, examples of recombination 
events involving the fusion of Alu and L1 repeats have not been reported.

Deletions extending from exon 2 to exon 7 are among the most common deletion mutations in the dystrophin gene (Muntoni et al. 1994; Yuge et al. 1999). However, the molecular mechanism underlying the generation of these deletions remains unknown. In this report, we describe the first example of a non-homologous recombination event between Alu and L1 repeats as a cause of a 430-kb deletion in the dystrophin gene that is responsible for a case of BMD.

\section{Subjects and methods}

Genomic DNA was extracted from whole blood drawn from a 6-year-old Japanese boy suffering from BMD, and from his family members. The boy was diagnosed with BMD on the basis of high serum creatine kinase (CK) levels, electromyogram analysis, and muscle biopsy. Both his mother and his older sister showed elevated serum CK levels. His maternal uncle was diagnosed with idiopathic dilated cardiomyopathy at the age of 21 , and underwent heart transplantation.

The presence of a deletion mutation in the dystrophin gene was examined in 19 deletion-prone exons that are clustered within two deletion hot spots and which were amplified by PCR, using primer sets described previously (Chamberlain et al. 1988; Beggs et al. 1990). In order to determine the borders of the deletion, the genomic region encompassing exons 2 or 7 was PCR amplified (Dwi Pramono et al. 2000) and dystrophin cDNA was analyzed as described before (Hagiwara et al. 1994). The breakpoints within introns 1 or 7 were examined by successive PCR amplification reactions, and four and five regions were sub- sequently amplified from introns 1 and 7, respectively (Fig. 1). In order to clone the breakpoint, PCR was carried out using primers that hybridized close to the breakpoints of the deletion: a forward primer in the sequence of intron 1 and a reverse primer complementary to the sequence of intron 7 . A fragment of more than $10 \mathrm{~kb}$ was amplified. This product was digested with the restriction enzyme SauIIIAI. Finally, sequences around the breakpoint were determined by sequencing the sub-cloned fragments. PCR was carried out essentially as described before (Narita et al. 1993). Primers used in this study are listed in Table 1. PCR-amplified products or digested fragments subcloned into pT7 blue T vector (Novagen, Madison, WI, USA) were sequenced using a Taq dye termination cycle sequencing kit (Perkin Elmer Applied Biosystems, Norwalk, CT, USA) with an automatic DNA sequencer (model ABI Prism 310 Genetic Analyzer; Perkin Elmer Applied Biosystems), as described previously (Surono et al. 1999).

\section{Results}

Nineteen deletion-prone exons of the dystrophin gene were examined in the index case, by PCR amplification. Exons located in the central deletion hot spot were amplified as normal. In the $5^{\prime}$ region, however, exons 3,4 , and 6 could not be amplified, whereas exons 1 and 8 could be amplified, suggesting the presence of a deletion spanning from exons 3 to 6 . In order to determine the border of the deletion, the flanking exons, 2 and 7, were examined. An amplification product corresponding to these two exons could not be obtained. Considering that exons 1 and 8 were present, the index case must have a deletion spanning from exons 2 to 7 (Fig. 1). Analysis of dystrophin cDNA prepared from the index case revealed that the $3^{\prime}$ end of exon 1 was joined

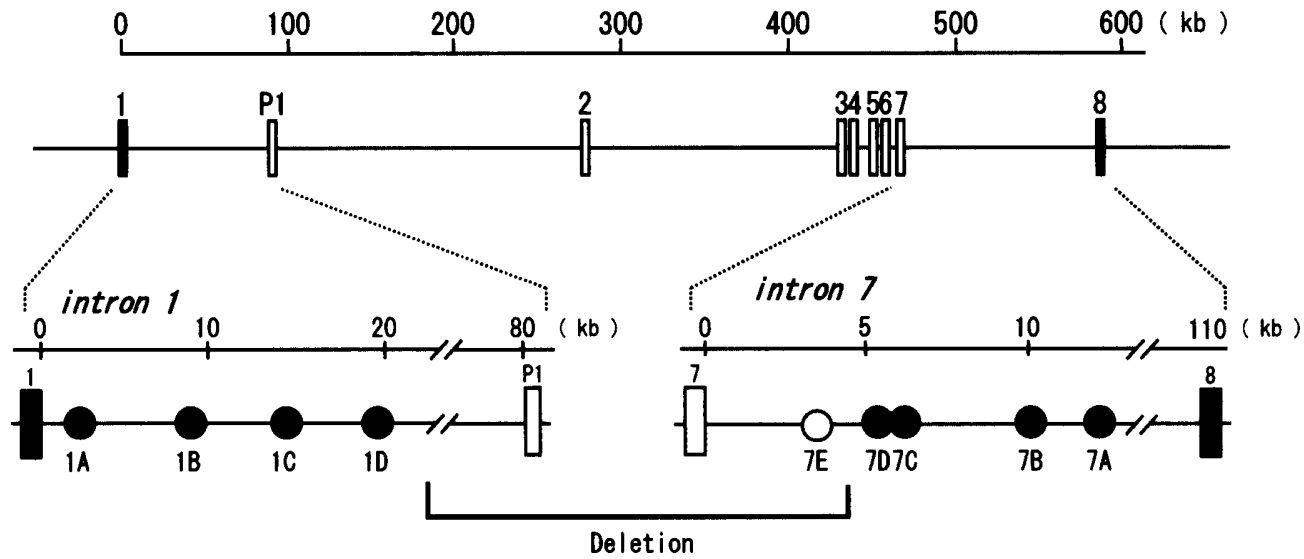

Fig. 1. Physical map of the $5^{\prime}$ region of the dystrophin gene. The deletion of the genomic region in the $5^{\prime}$ region of the dystrophin gene was examined by polymerase chain reaction (PCR) amplification, as indicated by boxes and circles. Numbers over each box indicate the exon number. $P 1$ indicates the P-promoter and the first exon region. Horizontal lines indicate introns. Filled and open boxes represent exons and deleted exons, respectively. Open and filled circles on intron se- quences represent deleted and retained regions, respectively. $1 \mathrm{~A}, 1 \mathrm{~B}$, $1 \mathrm{C}$, and $1 \mathrm{D}$ are located at nearly $1,8,16$, and $20 \mathrm{~kb}$ downstream from the exon 1 , respectively. $7 \mathrm{~A}, 7 \mathrm{~B}, 7 \mathrm{C}, 7 \mathrm{D}$, and $7 \mathrm{E}$ are $13,10,7,5$, and $4 \mathrm{~kb}$ downstream from exon 7 , respectively. Enlarged maps of introns 1 and 7 are shown below the full genomic map. Bracket represents a deletion region 
Table 1. List of primers prepared in this study

\begin{tabular}{lll}
\hline Fragment & Forward primer(f) & Reverse primer(r) \\
\hline 1A & GATGAATCCCAATGACTCAC & ACTAAATCAGGATTCCCCAG \\
1B & CTGTGAATATTACCAAAATG & CAGGTTAGCCCAGTTCTTAGCT \\
1C & CCAGTCTAACAACCTATTCATA & GATTGTCATGTCACATACAAGC \\
1D & CCTCACCTAGTACTAGCTTAGA & ACACCTATACTGCCCTAGTAAG \\
7A & TTCTGGGCAGTTGGAACCAG & AACAAATCAGCCCCTAGGGG \\
7B & GGCTGCAAATAAGATGAGGATTC & TTTGGAGAGTAGCCAACATCATG \\
7C & GAACTTGACCACTTGGCTCC & CCAGAGCTGAGCTCAAGTAC \\
7D & GGCTGCTGCCTTTCTTCAG & GGAGCCAAGTGGTCAAGTTC \\
7E & GTAGGTTGACGAGCCTGTAATC & GCGATAAGATGCTCTTTCAATTGG \\
7F & CCAGAAGACAGCCTTTTGCC & GGTAGGAGGGATACTATCGTG \\
\hline
\end{tabular}

directly to the $5^{\prime}$ end of exon 8 (data not shown), confirming the deletion of exons 2 to 7 .

Because deletions of exons 2 to 7 of the dystrophin gene have already been reported in several BMD cases in different ethnic group (Muntoni et al. 1994; Yuge et al. 1999), it was proposed that the deletion endpoint includes a genomic structure or element that predisposes this region to deletion. To examine this proposal, genomic sequences around the deletion endpoint were analyzed. First, the deletion endpoint intron 1 was defined more precisely. The Purkinje promoter/exon 1 (P-promoter) region, which is one of alternative promoters and is present about $80 \mathrm{~kb}$ downstream from exon 1 (Gorecki et al. 1992), was analyzed by PCR amplification. However, no amplified product was obtained, suggesting that the deletion endpoint is between exon 1 and P1 (Fig. 1). Subsequently, successive fragments of intron 1, starting from the $3^{\prime}$ end of exon 1 , were examined by PCR amplification. Using the available sequence of intron 1, we designed four sets of primers (1A, 1B, 1C, and 1D) (Fig. 1, Table 1). All four regions, located $1,8,16$, and $20 \mathrm{~kb}$ from the $5^{\prime}$ end of exon 1 , respectively, could be amplified from the patient's DNA, as well as from the control DNA (data not shown). Therefore, the deletion did not affect at least $20 \mathrm{~kb}$ of the $5^{\prime}$ region of intron 1 .

In order to narrow down the deletion endpoint in intron 7 , five regions, from $7 \mathrm{~A}$ to $7 \mathrm{E}$, in intron 7 were examined by PCR amplification. Regions 7A, 7B, 7C, and 7D, but not 7E, could be amplified (data not shown). These results indicated that the deletion endpoint is between regions 7D and $7 \mathrm{E}$, which are $5 \mathrm{~kb}$ and $4 \mathrm{~kb}$ downstream from the $3^{\prime}$ end of exon 7, respectively (Fig. 1).

Finally, we attempted to amplify the deletion endpoint using a primer set comprising a forward primer in intron 1 (1D f) and a reverse primer in intron 7 (7D r) (Table 1). A product of more than $10 \mathrm{~kb}$ was amplified (Fig. 2). A fragment of the same size was also amplified from the genomic DNA of the mother of the index case, who was considered to be a carrier (data not shown). This indicated that the amplified product was from the recombination region; therefore, the amplified product was subjected to sequence analysis, using restriction enzyme-digested fragments. In the fragment containing the breakpoint, the sequence of the $5^{\prime}$ portion of the fragment was completely homologous to the sequence of intron 1 (Genbank AL049643), while the
Fig. 2. PCR amplified product spanning from intron 1 to 7 . An amplified fragment of more than $10 \mathrm{~kb}$ was obtained from the patient's genomic DNA $(P)$, but not from control $(C)$, when primers on intron 1 (1D f) and 7 (7D r) were used. $M$ represents a size marker, which is HincIIdigested $\phi X 174$ phage DNA (Toyobo, Osaka, Japan)

remaining $3^{\prime}$ portion of the fragment was completely identical to the sequence of intron 7 (Genbank U60822) (Fig. 3). These results indicated that the deletion end points were $25 \mathrm{~kb}$ downstream from the $3^{\prime}$ end of muscle exon 1 and $4.5 \mathrm{~kb}$ downstream from the $3^{\prime}$ end of exon 7 (Fig. 1). The size of the deletion was calculated to be $430 \mathrm{~kb}$, according to the approximate mapping data (Coffey et al. 1992).

One $\mathrm{kb}$ of sequences on each side of the deletion breakpoints in intron 1 and 7 were then examined (Fig. 4). It was found that $296 \mathrm{bp}$ encompassing the deletion endpoint of intron 1 was homologous to the $\mathrm{Sg}$ member of the Alu repeat family. The $3^{\prime}$ breakpoint in intron 7 was located within the sequence of open reading frame 1 of an L1 element. However, the DNA sequences flanking the $5^{\prime}$ and $3^{\prime}$ breakpoints in the wild-type dystrophin gene are not homologous (Fig. 3). Thus, we concluded that a nonhomologous Alu-L1 recombination event is likely to be responsible for this deletion. Significantly, the 26-bp core sequence of Alu repeats that has been reported to trigger recombination and to cause the frequent involvement of Alu elements in gene rearrangements (Rudiger et al. 1995) was identified 34nt upstream from the breakpoint (Fig. 3).

Possible matrix attachment region (MAR) sites in introns 1 and 7 were also analyzed, using the MAR-Finder program of Singh et al. (1997). The region around the breakpoints included several MAR motifs (Fig. 4). Secondary structure analysis (Zuker 1989) revealed that the breakpoint in intron 1 was in the middle of the hairpinloop structure formed by two complementary sequences: CAGGATG upstream of the breakpoint and the down- 
stream complementary CATCCTG (Fig. 3). Remarkably, the sequence CAGG that was originally found in a deletion hot spot in the murine MHC gene cluster (Steinmetz et al. 1987), and the oppositely orientated CCTG sequence that was frequently observed in deletion hot spots of the globin
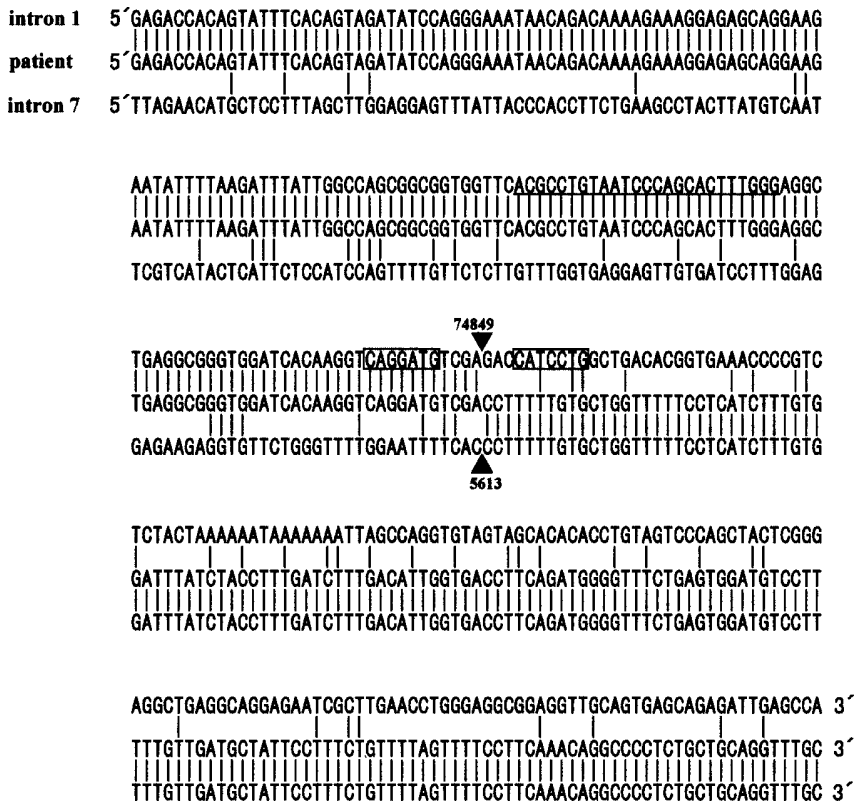

Fig. 3. DNA sequence spanning the deletion junction with the corresponding intron sequences. The intron 1 sequence (top line) (Genbank AL049643), the sequence containing the breakpoint (middle line), and the intron 7 sequence (bottom line) (Genbank, U60822) are aligned. The bars between the sequences indicate positions at which they are identical. Filled arrowheads indicate the deletion breakpoint. Numbers over and under the arrowheads indicate the nucleotide numbers of the end of the homologous region to intron 1 (Genbank AL0496439) and intron 7 (Genbank, U60822), respectively. The 26-bp core sequence of Alu repeats is underlined. The boxed segments indicate regions containing the hairpin structure in which two 7-bp stretches of DNA on the same strand are complementary genes (Krawczak and Cooper 1991) were identified in these complementary sequences.

In addition, the sequence TTTT, the putative polymerase mutation hot spot that is frequently found around deletion breakpoints (Krawczak and Cooper 1991), was observed several times near the $3^{\prime}$ breakpoint in intron 7 . Finally, sequences homologous to the topoisomerase II binding and cleavage consensus sequences were found in both introns 1 and 7, especially near the breakpoint of intron 1 . However, a topoisomerase I cleavage site was not identified near the recombination point (Fig. 4).

\section{Discussion}

Although the approximately 3000-kb dystrophin gene is a large target for mutations, the mechanisms that promote the observed high frequency of spontaneous mutations in this gene are not well understood. In this report, we propose that the molecular basis of a deletion spanning exons 2 to 7 of the dystrophin gene is a non-homologous recombination event between an Alu repeat in intron 1 and an L1 repeat in intron 7 (Fig. 4). The data demonstrate that deletion mutations are not necessarily produced by homologous DNA misalignments, as noted previously (McNaughton et al. 1998).

Southern blot analysis indicated the presence of two deletion hot spots in the dystrophin gene (Koenig et al. 1989). Den Dunnen et al. (1989) proposed that two of the longer introns, 7 and 44, might contain factors that predispose them to deletion. Previously, we pointed out that one small deletion in the dystrophin gene was caused by a palindromic structure near the deletion junction (Matsuo et al. 1990). However, molecular analyses of the endpoints of large deletions have been performed in only a limited number of cases. Alu or L1 repeats have been identified at either end of several deletions (Ehrenpreis et al. 1991; Love et al. 1991; Baldrich et al. 1992; Pizzuti et al. 1992; McNaughton et al.

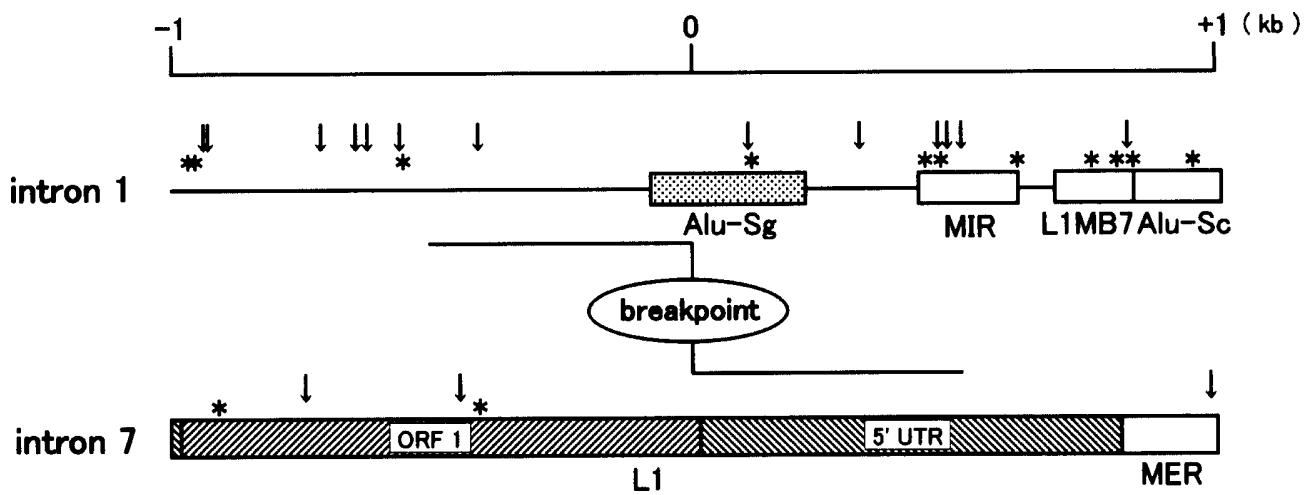

Fig. 4. Physical DNA map of the region around the deletion breakpoint. The organization of $2 \mathrm{~kb}$ of intron 1 and intron 7 is shown. Lines indicate introns. Boxes represent the repetitive genomic sequences as marked below. MIR and MER represent mammalian-wide interspersed repeats and medium reiteration repeats, respectively. Dotted and shaded boxes are the Alu-Sg in intron 1 and the L1 repeat in intron 7 that are joined together. $O R F 1$ and $5^{\prime} U T R$ represent open reading frame 1 and the $5^{\prime}$ untranslational region of L1 repeat, respectively. Vertical arrows indicate topoisomerase II recognition sites (GTNWAYATTNATNNR). Asterisks show matrix attachment region (MAR) motifs (ATATTT) 
1998), suggesting that Alu or L1 repeats could be factors that predispose the dystrophin gene to instability. The large number of Alu elements within the human genome provides abundant opportunities for unequal homologous recombination events between Alu repeats, resulting in human diseases (Kornreich et al. 1990; Burwinkel and Kilimann 1998; Deininger and Batzer 1999). However, only one example of Alu-Alu recombination has been reported in the dystrophin gene, the deletion of exons 6 to 7 (McNaughton et al. 1998).

The other major repeat unit in the human genome is the L1 element. Unequal homologous recombination events between two neighboring L1 sequences are an established cause of genome instability (Burwinkel and Kilimann 1998), and L1 elements frequently contribute to recombination by diverse mechanisms (Segal et al. 1999). Here, we report the first example of non-homologous recombination between Alu and L1 elements.

Chromatin structure may bring distant termini close together as chromatin loops that span about 50 to $100 \mathrm{~kb}$ attach to the fibers of the chromosomal scaffold at MARs. It has been proposed that four large deletions arose in the human beta-globin gene cluster from the loss of integral numbers of chromatin loops anchored to the nuclear matrix during DNA replication (Vanin et al. 1983). In the present study, the presence of many MAR motifs near the breakpoint (Fig. 4) suggested that the 430-kb deletion may have involved at least four chromatin loops. The physical conformation of this region of the dystrophin gene may be an important factor in the etiology of the deletions (McNaughton et al. 1997). Because we identified several putative topoisomerase II consensus sequences in both introns 1 and 7 (Fig. 4), we suppose that this enzyme was instrumental in creating the chromatid exchange events. Supporting this supposition, a topoisomerase II consensus sequence has been identified in several breakpoints of nonhomologous recombination (Hu et al. 1991; Krawczak and Cooper 1991; Blok et al. 1995).

It has been proposed that deletions are generated by the formation of a hairpin loop structure in a single strand of DNA, followed by enzymatic degradation at unpaired regions within the loop (Robinson et al. 1997). Furthermore, it has been proposed that the sequences on either side of the deletion must have a high degree of intra-strand base complementarity. In apparent agreement with this, the 7-bp complementary sequences that flank the breakpoint in intron 1 in the deletion studied here may be one factor that predisposes this region to recombination (Fig. 3).

Intron 7 of the human dystrophin gene is unusually large $(110 \mathrm{~kb})$, and a substantial number of deletion endpoints have been identified within it (McNaughton et al. 1997). Defined deletion endpoints are dispersed throughout intron 7 , including several at different sites within $\mathrm{L} 1$ repeats. The L1 sequence in this report, which is near the $5^{\prime}$ end of intron 7 (Fig. 1) is different from those described previously.

Because we could not identify any single cause for the recombination event described here, we propose that it is generated by a combination of the above-mentioned sequence characteristics of the two introns that are involved.
Acknowledgments We thank Dr. A. Pugsley for advice and for critically reading the manuscript. We acknowledge Ms. N. Kageyama for secretarial help. This work was supported by grants from the Ministry of Education, Science, and Culture of Japan, and a Research Grant (8A-1) for Nervous and Mental Disorders, from the Ministry of Health and Welfare of Japan.

\section{References}

Baldrich K, Baldrich M, Monaco AP, Muller CR (1992) Replication errors may contribute to the generation of large deletions and duplications in the dystrophin gene. Hum Mutat 1:280-287

Beggs AH, Koenig M, Boyce FM, Kunkel LM (1990) Detection of 98\% of DMD/BMD gene deletions by polymerase chain reaction. Hum Genet 86:45-48

Blok RB, Thorburn DR, Thompson GN, Dahl HH (1995) A topoisomerase II cleavage site is associated with a novel mitochondrial DNA deletion. Hum Genet 95:75-81

Burwinkel B, Kilimann MW (1998) Unequal homologous recombination between LINE-1 elements as a mutational mechanism in human genetic disease. J Mol Biol 277:513-517

Chamberlain JS, Gibbs RA, Ranier JE, Nguyen PN, Caskey CT (1988) Deletion screening of the Duchenne muscular dystrophy locus via multiplex DNA amplification. Nucleic Acids Res 16:1114111156

Coffey AJ, Roberts RG, Green ED, Cole CG, Butler R, Anand R, Giannelli F, Bentley DR (1992) Construction of a 2.6-Mb contig in yeast artificial chromosomes spanning the human dystrophin gene using an STS-based approach. Genomics 12:474-484

Deininger PL, Batzer MA (1999) Alu repeats and human disease. Mol Genet Metab 67:183-193

Den Dunnen JT, Grootscholten PM, Bakker E, Blonden LA, Ginjaar HB, Wapenaar MC, van Paassen HM, van Broeckhoven C, Pearson PL, van Ommen (1989) Topography of the Duchenne muscular dystrophy (DMD) gene: FIGE and cDNA analysis of 194 cases reveals 115 deletions and 13 duplications. Am J Hum Genet 45:835847

Drechsler M, Royer-Pokora B (1996) A LINE element is present at the site of a $300-\mathrm{kb}$ deletion starting in intron 10 of the PAX6 gene in a case of familial aniridia. Hum Genet 98:297-303

Dwi Pramono ZA, Takeshima Y, Surono A, Ishida T, Matsuo M (2000) A novel cryptic exon in intron 2 of the human dystrophin gene evolved from an intron by acquiring consensus sequences for splicing at different stages of anthropoid evolution. Biochem Biophys Res Commun 267:321-328

Ehrenpreis J, Hillers M, Junkes B, Pfordt M, Schwinger E, Vosberg H-P (1991) Analysis of a dystrophin gene deletion by amplification of mRNA isolated from DMD myocytes cultured in vitro. Genomics 10:551-557

Forrest SM, Cross GS, Flint T, Speer A, Robson KJH, Davies KE (1988) Further studies of gene deletions that cause Duchenne and Becker muscular dystrophies. Genomics 2:109-114

Gorecki DC, Monaco AP, Derry JMJ, Walker AP, Barnard EA, Barnard PJ (1992) Expression of four alternative dystrophin transcripts in brain regions regulated by different promoters. Hum Mol Genet 1:505-510

Hagiwara Y, Nishio H, Kitoh Y, Takeshima Y, Narita N, Wada H, Yokoyama M, Nakamura H, Matsuo M (1994) A novel point mutation $(\mathrm{G}-1$ to $\mathrm{T})$ in a $5^{\prime}$ splice donor site of intron 13 of the dystrophin gene results in exon skipping and is responsible for Becker muscular dystrophy. Am J Hum Genet 54:53-61

$\mathrm{Hu}$ XY, Ray PN, Worton RG (1991) Mechanisms of tandem duplication in the Duchenne muscular dystrophy gene include both homologous and nonhomologous intrachromosomal recombination. EMBO J 10:2471-2477

Koenig M, Beggs AH, Moyer M, Scherpf S, Heindrich K, Bettecken T, Meng G, Muller CR, Lindlof M, Kaariainen H, de la Chapelle A, Kiuru A, Savontaus M-L, Gilgenkrantz H, Recan D, Chelly J, Kaplan J-C, Covone AE, Archidiacono N, Romeo G, Liecht-Gallati S, Schneider V, Braga S, Moser H, Darras BT, Murphy $\mathrm{P}$, Francke U, Chen JD, Morgan G, Denton M, Greenberg CR, Wrogemann K, Blonden LAJ, van Paassen HMB, van Ommen GJB, Kunkel LM (1989) The molecular basis for Duchenne versus Becker 
muscular dystrophy: correlation of severity with type of deletion. Am J Hum Genet 45:498-506

Koenig M, Hoffman EP, Bertelson CJ, Monaco AP, Feener C, Kunkel LM (1987) Complete cloning of the Duchenne muscular dystrophy (DMD) cDNA and preliminary genomic organization of the $D M D$ gene in normal and affected individuals. Cell 50:509-517

Kornreich R, Bishop DF, Desnick RJ (1990) Alpha-galactosidase A gene rearrangements causing Fabry disease. Identification of short direct repeats at breakpoints in an Alu-rich gene. J Biol Chem 265:9319-9326

Krawczak M, Cooper D (1991) Gene deletions causing human genetic disease: mechanisms of mutagenesis and the role of the local DNA sequence environment. Hum Genet 86:425-441

Kumatori A, Faizunnessa NN, Suzuki S, Moriuchi T, Kurozumi H, Nakamura M (1998) Nonhomologous recombination between the cytochrome b558 heavy chain gene $(C Y B B)$ and LINE-1 causes an $\mathrm{X}$-linked chronic granulomatous disease. Genomics 53:123-128

Love D, England S, Speer A, Marsden R, Bloomfield J, Roche A, Gross G, Mountford RC, Smith TJ, Davies KE (1991) Sequences of junction fragments in the deletion-prone region of the dystrophin gene. Genomics 10:57-67

Matsuo M, Masumura T, Nakajima T, Kitoh Y, Takumi T, Nishio H, Koga J, Nakamura H (1990) A very small frame-shifting deletion within exon 19 of the Duchenne muscular dystrophy gene. Biochem Biophys Res Commun 170:963-967

McNaughton JC, Hughes G, Jones WA, Stockwell PA, Klamut HJ, Petersen GB (1997) The evolution of an intron: analysis of a long, deletion-prone intron in the human dystrophin gene. Genomics 40:294-304

McNaughton JC, Cockburn DJ, Hughes G, Jones WA, Laing NG, Ray PN, Stockwell PA, Petersen GB (1998) Is gene deletion in eukaryotes sequence-dependent? A study of 9 deletion junctions and 19 other deletion breakpoints in intron 7 of the human dystrophin gene. Gene 222:41-51

Morgan NV, Tipping AJ, Joenje H, Mathew CG (1999) High frequency of large intragenic deletions in the Fanconi anemia group A gene. Am J Hum Genet 65:1330-1341

Moser H (1984) Duchenne muscular dystrophy: pathogenetic aspects and genetic prevention. Hum Genet 66:17-41

Muntoni F, Gobbi P, Sewry C, Sherratt T, Taylor J, Sandhu SK, Abbs S, Roberts R, Hodgson SV, Bobrow M, Dubowitz V (1994) Deletions in the $5^{\prime}$ region of dystrophin and resulting phenotypes. J Med Genet 31:843-847
Narita N, Nishio H, Kitoh Y, Ishikawa Y, Ishikawa Y, Minami R, Nakamura H, Matsuo M (1993) Insertion of a $5^{\prime}$ truncated L1 element into the $3^{\prime}$ end of exon 44 of the dystrophin gene resulted in skipping of the exon during splicing in a case of Duchenne muscular dystrophy. J Clin Invest 91:1862-1867

Pizzuti A, Pieretti M, Fenwick RG, Gibbs RA, Caskey CT (1992) A transposon-like element in the deletion-prone region of the dystrophin gene. Genomics 13:594-600

Robinson DO, Bunyan DJ, Gabb HA, Temple IK, Yau SC (1997) A small intraexonic deletion within the dystrophin gene suggests a possible mechanism of mutagenesis. Hum Genet 99:658-662

Rudiger NS, Gregersen N, Kielland-Brandt MC (1995) One short well conserved region of Alu-sequences is involved in human gene rearrangements and has homology with prokaryotic chi. Nucleic Acids Res 23:256-260

Segal Y, Peissel B, Renieri A, de Marchi M, Ballabio A, Pei Y, Zhou J (1999) LINE-1 elements at the sites of molecular rearrangements in Alport syndrome-diffuse leiomyomatosis. Am J Hum Genet 64:6269

Singh GB, Kramer JA, Krawetz SA (1997) Mathematical model to predict regions of chromatin attachment to the nuclear matrix. Nucleic Acids Res 25:1419-1425

Smit AF (1996) The origin of interspersed repeats in the human genome. Curr Opin Genet Dev 6:743-748

Steinmetz M, Uematsu Y, Lindahl K (1987) Hotspots of homologous recombination in mammalian genomes. Trends Genet 3:7-10

Surono A, Takeshima Y, Wibawa T, Ikezawa M, Nonaka I, Matsuo M (1999) Circular dystrophin RNAs consisting of exons that were skipped by alternative splicing. Hum Mol Genet 8:493-500

Vanin EF, Henthorn PS, Kioussis D, Grosveld F, Smithies O (1983) Unexpected relationships between four large deletions in the human beta- globin gene cluster. Cell 35:701-709

Yuge L, Hui L, Bingdi X (1999) Detection of gene deletions in Chinese patients with Duchenne/Becker muscular dystrophy using cDNA probes and the polymerase chain reaction method. Life Sci 65:863869

Zhang XQ, Zhang JW (1998) The 3' breakpoint of the Yunnanese $\left({ }^{\mathrm{A}} \gamma \delta \beta\right)^{0}$-thalassemia deletion lies in an L1 family sequence: implications for the mechanism of deletion and the reactivation of the $\gamma$ globin gene. Hum Genet 103:90-95

Zuker M (1989) Computer prediction of RNA structure. In: Dahlberg J, Abelson J (eds) Methods in enzymology. Vol. 180. Academic, San Diego, pp 263-288 